\title{
Parametric Design Method Based on Topological and Geometrical Constraints
}

\author{
Masatake Higashi, Hiroki Senga, Atsuhide Nakamura and Mamoru Hosaka
}

Toyota Technological Institute, 2-12-1, Hisakata, Tempaku-ku, Nagoya, 468-8511 JAPAN

\begin{abstract}
This paper proposes a parametric design method which realizes a product model in the computer according to the designer's intent and modifies its shape directly according to the change of dimensional parameters. The designer's intent is represented as topological and geometrical constraints, which the designer specifies explicitly or implicitly with the shape properties, position and size of a feature. Direct modification of features is realized with a swept volume generated locally instead of the reevaluation of a history of feature operations which is used in the current parametric modeling systems. The product model composed of a feature model and a geometric model is expressed with face-based representation and processed symbolically in an algebraic computation system. The algorithms of the feature generation and modification are described and their examples are shown. Efficient and unambiguous parametric modeling have been realized by detecting and correcting inconsistency between the feature model and geometric model.
\end{abstract}

Key words: Parametric design, Feature modeling, Geometrical constraint, Topological constraint

\section{INTRODUCTION}

Feature modeling has been introduced to integrate digital processes of product development through the product life cycle (Shah \& Mäntylä, 1995). Here, a feature is defined as a shape which represents a characteristic shape associated with the designer's intent concerning product functions and methods or processes for its manufacturing. In the current commercial feature modeling systems, features are generated mainly by sweeping operations for two-dimensional shapes with dimensional parameters, and 
history-based modeling functions are used to realize parametric design. As a result, the designer's intent is indirectly represented by a sequence of operation commands, and it is not directly described as constraints in the product model as shown in the engineering drawings. Therefore, the historybased approach needs so much computational cost to modify the shape, while it has a topological naming problem where it cannot identify the geometrical entities when they are deleted or combined with other elements.

To resolve the above problems, many researchers proposed methods. Their focuses are divided into two categories. One is on persistent naming of topological elements and the other is on validity of feature semantics. In the first category, they want to specify topological elements such as vertex, edge and face without ambiguity in the reevaluation of feature operations. Kripac (1995) proposed a solution for a practical modeling system, and Hoffmann and his group (Hoffmann et al, 1993, 1998, Chen et al, 1995-1, 1995-2, 1995-3; Capoyleas, 1996) tried to solve this problem based on the textual representation which was proposed for editable and translative feature modeling independent of a kernel modeling system. However, since it is necessary to classify the patterns of topological modifications, the algorithms become complicated but not perfect.

On the other hand, in the second category, they want to maintain validity or semantics in the feature modeling. By combination with a cellular model (Rossignac, 1990; Bidarra et al, 1998, 1999), or consistent updates between CSG and B-rep (Raghothama et al, 1999), interactions of features are described and manipulated consistently. They can check validity incrementally, but cannot modify the shape directly according to the change of parameters.

Different from above approaches, we propose a feature modeling method which deals with topological and geometrical constraints of features as the designer's intent which the designer specifies explicitly or implicitly with the shape properties, position and size of a feature. We modify the product model directly from the specified dimension value instead of the reevaluation of a history of feature operations, and obtain consistent constraints in the model without intermediate descriptions such as a cellular model. The characteristics of the method are described as follows.

Symbolical face-based representation is introduced for uniformly treating a feature model (constraints information) and a geometric model (shape description).

Topological constraints are introduced for treating them independently from geometrical constraints. A product shape without dimensions can be generated only from topological constraints, and an individual face can be constrained with a dimensional value as on the engineering drawing. 
An influence region for the dimensional change of a specified face is calculated as a swept volume using its adjacent faces. The volume is utilized for detecting feature interference and modifying the topological structure.

The result of interference calculation for the influence region, which includes information such as deleted, divided, combined and newly generated faces, maintains consistent feature types (validity) and geometrical constraints.

In this paper, we describe a feature model composed of topological and geometrical constraints, a geometric model expressed by Face-based representation, and generation method of topological constraints according to the user indication of feature attachment in Section 2. Next, we describe geometrical constraints which prescribe geometric relations among the topological elements in Section 3. Then we indicate how the constraints are maintained in Section 4.

\section{FEATURE MODEL AND TOPOLOGICAL CONSTRAINTS}

\subsection{Representation of feature model and geometric model}

A product model is composed of a feature model and a geometric model as shown in Fig. 1. A feature model consists of a feature table, geometrical constraints and a dimension table, while a geometric model is composed of a face connection table and a face data table. The feature table includes a name, a type, an attribute and component faces of a feature, along with topological constraints which indicate coincident faces and their direction attributes. According to the indication of feature attachments by a designer, a feature model is deduced automatically as topological and geometrical constraints, and then a geometric model is generated using the constraints. When parameter values are changed by the designer, the validity of the feature model is maintained according to the result of interference calculation in the geometric model.

We define a basic feature as a region where edges have the same convex/concave property. We can generate basic features such as slot (open/ close), step (open/close), hole (through/blind), pocket, rib and boss, by designating coincident faces for a hexahedron before their geometry is determined, if there is no interference of features. The faces of the hexahedron are not necessarily planes and the hexahedron itself can be 
replaced with a convex cell which is a basic cell for a generic feature. For example, for a hole, a cylinder is used.

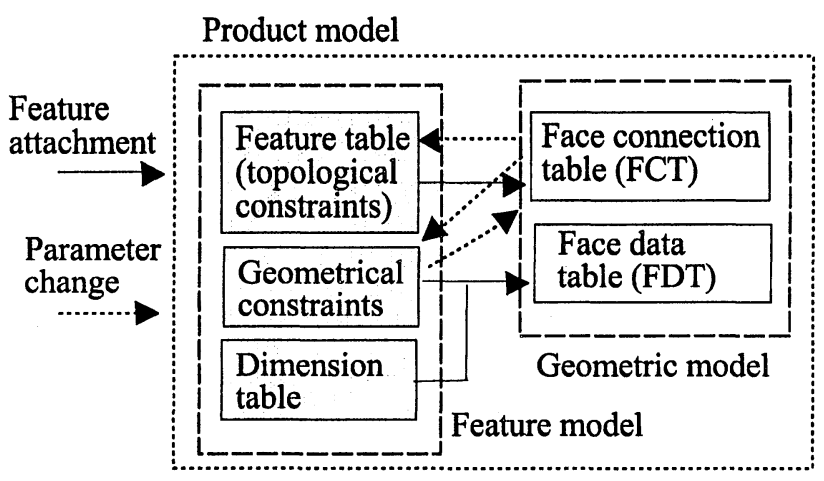

Figure 1: Structure of a product model

The feature model is generated from the designer's indication: how features are attached to a product. It is described by a feature attribute (add / $s u b$ ) and how faces correspond between a feature and a product. The list of pairs of coincident (corresponding) faces is described as topological constraints in a feature model. The topological constraints also include attributes for coincident directions of faces (same, opposite, deletion by interference: $1,-1,0)$ which are automatically deduced in the feature attachment or feature interaction and which determine the convexity or concavity of the feature boundary. If the attributes are opposite and deletion which is derived when the feature face is deleted, the boundary of the feature has an opposite property of convex/concave against that of the inner edges and they are treated similarly in the feature classification. If the attribute is same, faces of a product and a feature are shared without a boundary.

The topology of a geometric model is generated from the feature model and is described by the Face-based representation (F-Rep) (Hosaka \& Sata, 1994; Higashi et al, 1995). The F-Rep is composed of a face connection table, a face data table and constraint relations for faces. The face connection table (FCT) consists of names (IDs) of faces and their adjacentfaces lists (AFL). An AFL is a sequence of face IDs ordered anti-clockwise around the face looked at from the outside and composes a boundary face loop. Fig. 2 shows a FCT of a hexahedron with six faces: Front, Right, Back, Left, $U p$ and Down. The face data table (FDT) includes equations for faces, which are described with a unit normal vector and a distance from the origin for each plane. 


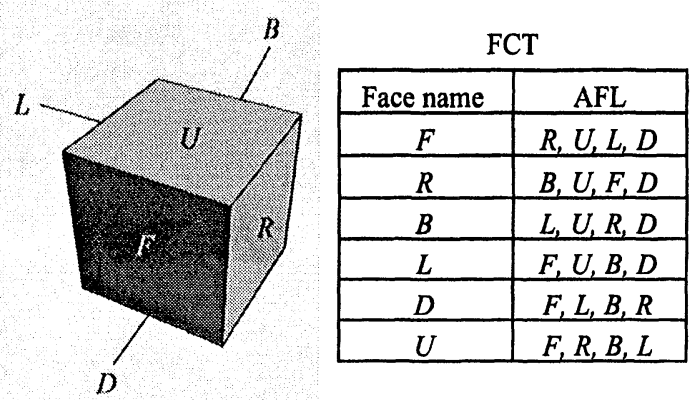

Figure 2: F-Rep for a hexahedron to be used for feature creation

\subsection{Topological constraints generated by feature attachment}

The algorithms of the feature attachment are divided into two types: inside attachment and multiple-faces coincidence. Here, additive and subtractive features are uniformly processed by reversing the order of the AFLs for the subtractive features. The inside attachment is used to generate features such as a boss, a pocket, a hole and a bridge which have no interference of face loops with other faces. The AFL of the specified face in the feature is simply added to that of the product as multiple loops, and the AFLs of the other feature faces are added to the FCT replacing the names of coincident faces with those of the product. On the other hand, such features as a slot are generated for multiple faces to be coincident. The algorithm symbolically generates the topology of the product as follows. For each pair of coincident faces, it deletes the other coincident faces included in the AFL, separates the list there, and then combines the corresponding divided list of the feature into that of the product face adding the adjacent coincident faces.

When a number of separated lists is more than one, the face is divided corresponding to the number. This is done uniformly in spite of the number of coincident faces, the number of generated face loops and convexity/concavity of edges of the feature boundary.

Fig. 3 shows an example of generating a closed step, specifying coincidences of three pairs of faces: $(1, F),(2, R)$ and $(6, U)$. The AFL of face " 1 " is made into $(4,5)$ from $(2,6,4,5)$ by deleting coincident faces " 2 " and " 6 ", added $(D, L)$ with deleted part $(2,6)$, and modified into $(D, L, 6,4$, $5,2)$. The AFLs of faces " 2 " and " 6 " are treated similarly, and the AFLs of 
faces " $B$ ", " $L$ " and " $D$ " are added to the FCT replacing the coincident faces with those of the product.

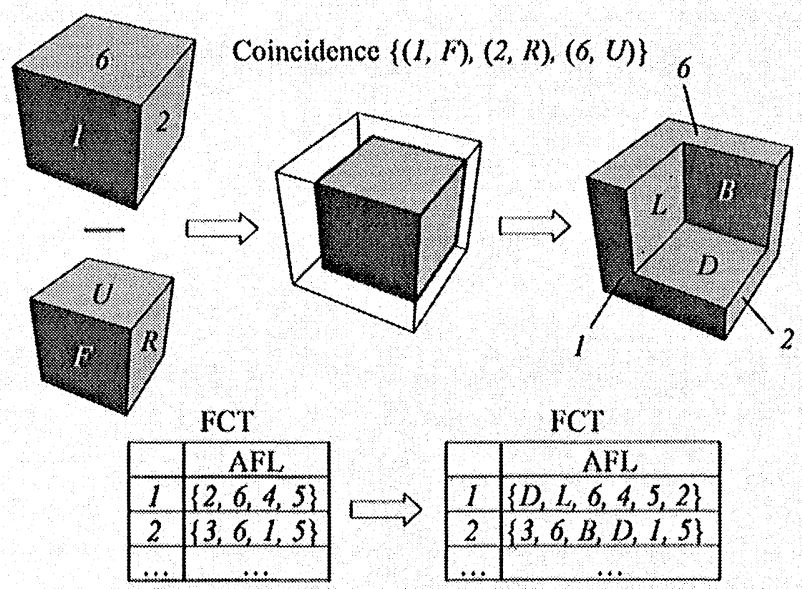

Figure 3: Feature generation by specifying coincident faces

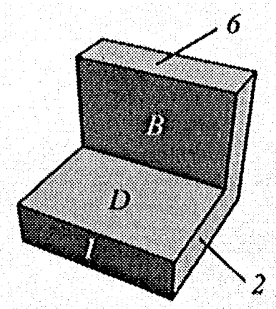

(a)

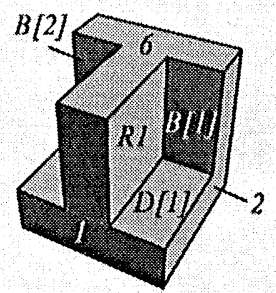

(d)

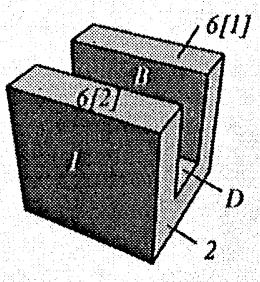

(b)

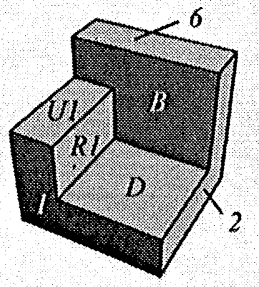

(c)

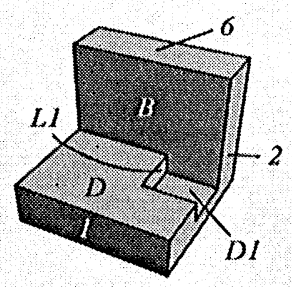

(c)

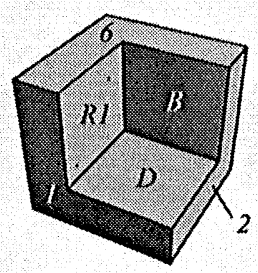

(f)

Figure 4: Patterns of feature generation according to coincident faces.

(a) Sub, $\{(1, F),(2, R),(4, L),(6, U)),\{-1,-1,-1,-1\}$

(b) $S u b,\{(2, R),(4, L),(6, U)\},\{-1,-1,-1\}$

(c) $\operatorname{Sub},\{(D, U 1),(2, R 1),(B, B 1)\},\{-1,-1,1\}$

(d) $\operatorname{Add},\{(D, D 1),(B, B 1),(F, F 1),(6, U 1)\},\{-1,-1,1,1\}$

(e) Add, $\{(D, D 1),(B, B 1),(1, F 1),(4, L 1)\},\{-1,-1,1,1\}$

(f) $\operatorname{Add},\{(D, D 1),(B, B 1),(1, F 1),(4, L 1),(6, U 1)\},\{-1,-1,1,1,1\}$ 
Fig. 4 shows several patterns generated uniformly by the algorithm of feature attachments. The caption indicates topological constraints generated: feature attribute, pairs of coincident faces and their direction attributes. Upper figures show subtractive features and lower ones show additive features. From (c) to (e), a feature is attached to the shape (a). The face with parentheses [ ] means that it is divided into multiple faces, and helps persistent naming of the element by directly indicating the original face of the divided ones.

When a designer wants to specify a shape intersecting a feature with a product, he can indicate which edges intersect with which faces. This indication determines the topology of the shape. For this purpose, we introduce a symbol of intersection point as a constraint, which expresses that an edge intersects with a face with three face names: two faces for an edge and one for an intersecting face. The names of faces are ordered as an anticlockwise sequence around the intersection point when we look at it from the outside, then the symbol also expresses the topological constraints of the intersection graph, that is the connectivity of the intersection points. Fig. 5 shows an example of an interfered boss. Using the six intersection points which are derived from the user indication, the feature is attached to a block.

However, when he wants that some part of the feature has fixed geometry, for example, the top face of a boss is fixed and the other part (the side faces of the boss) is generated intersecting it with the product, we calculate intersection points of the edges and generate the topology automatically using their intersection point symbols. This function is also used when he makes a product, part by part, and then combines them by Boolean set operations.

$(6, B, R),(6, L, B),(I, L, G),(I, D, L)\}$

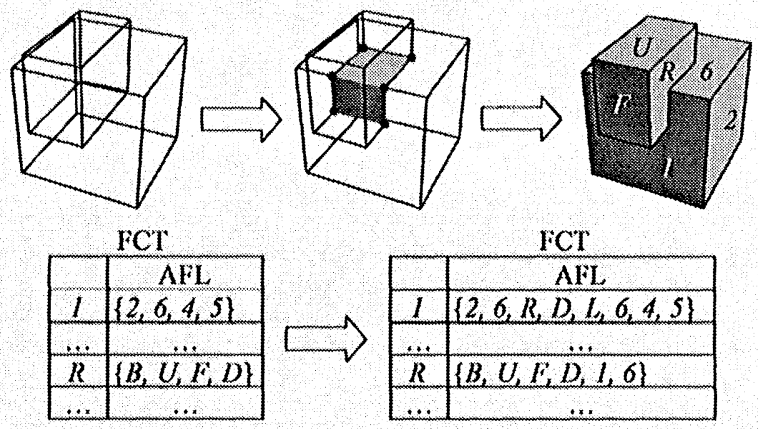

Figure 5: Feature generation by intersection point symbols 
Once the intersection-point symbols are determined, the intersection graph is generated by connecting them symbolically according to their connectivity, and the face loops are calculated using the graph. The algorithm is the same to that used in the Boolean set operation (Nakamura et al, 1997). For example, when face $A$ with a face loop $(B, C$, $D, E)$ intersects with faces $X$, $Y$ at intersection points $(A, B$, $X)$ and $(A, Y, C)$, the intersection generated newly is constrained so that it comes in $A$ from edge $(A, B)$ and goes out to edge $(A, C)$.

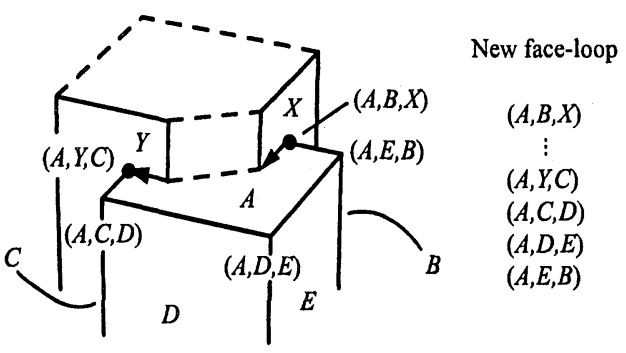
Then a new face loop is deduced as $(B, X, \ldots, Y, C, D, E)$. We obtain the loop as follows. First, we pick out intersection points and vertices with face $A$ in their symbols, and circulate their symbols so that $A$ becomes at the top. Then we obtain the face loop by tracing the faces so that the last face in the name is the second of the next symbol until we come back to the start of the face loop, which is the coming-in intersection point.

\section{GEOMETRICAL CONSTRAINTS}

To realize parametric design, we symbolically describe geometrical constraints in a geometrical-constraints list, a dependent-faces tree (DFT) and face equations in the FDT, which are processed consistently for the change of the parameter. These expressions are deduced from the dimensions among geometric elements specified by the designer similarly as done on the engineering drawings. Dimensions which the designer specifies are indicated to faces, edges and vertices, and they are treated as constraints for faces. A face is specified directly when it is parallel to the datum plane or it is rotated around the given axis from the datum plane, but auxiliary planes are used to fix a vertex or edge. The relations among the faces which are specified with dimensional values are represented as a tree structure (DFT): a parent is the datum face and a child is the referenced face. They express face dependency and are used to detect faces which are under or over constrained when their parent is deleted or combined. 


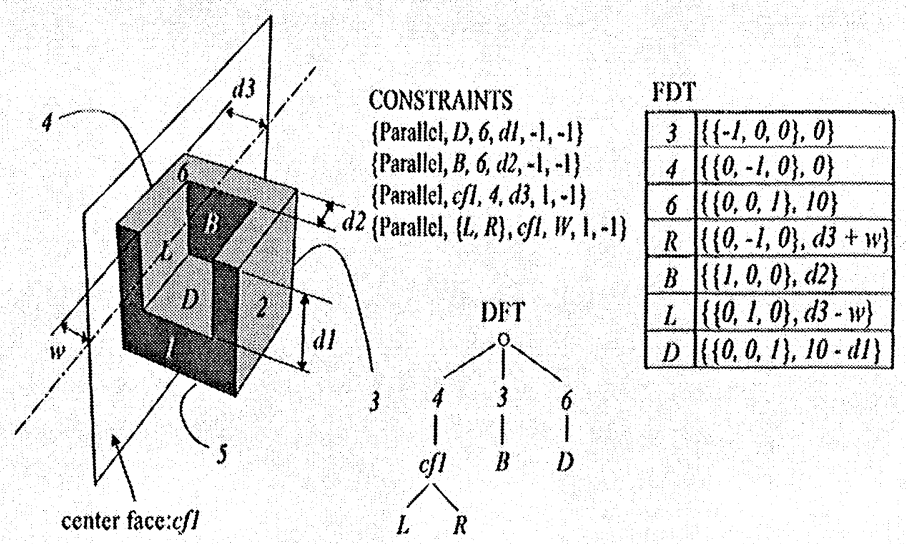

Figure 7: Expression of face equations with dimensional variables in FDT

The specified distances and angles are expressed in the FDT as variables in the face equations using Mathematica ${ }^{1}$ to change them freely. A centerline in the drawing is represented with a center face which is defined with an auxiliary plane and whose distance from a product face is specified. Then both side faces which are specified with a width parameter are determined with the parallel constraint from the center face. When all the component faces of the feature are specified, the geometry of the geometric model is fixed and checked whether the feature interferes with the other part of the product. When the designer changes the value of a parameter, the system changes the corresponding value in the parameter table and substitutes the variable in the FDT with the value. Generation of the modified shape can be executed at once if there is no topological modification, because the vertex coordinates are also expressed with these symbols in Mathematica.

Fig. 7 shows examples of face equations specified by dimensions for positions and a size: $d 1, d 2, d 3$ and $W$. A DFT and a FDT are generated according to the geometrical constraints which indicate type of constraint, object face, datum face, parameter variable, direction of face and direction of distance or angle as shown in the figure. The equations of Faces $R, B, L$ and $D$ are expressed using the equations of the datum faces and dimensional variables.

${ }^{1}$ Wolfram Research Inc. 


\section{PARAMETRIC DESIGN AND MAINTENANCE OF CONSISTENT CONSTRAINTS}

\subsection{Interference calculation using influence region}

Since all the geometry of faces and vertices of the shape is represented symbolically with dimensional parameters of features, the shape modification by changing the parameters is simple and quick if there is no feature interference. Hence the problem is how to detect this. We introduce an influence region for a parameter change. It is a region which is generated by sweeping the specified face and composing a cell with the adjacent faces. Here, the direction of the movement of the face determines the property of the cell, $A d d$ or $S u b$, and the convexity of the edges between the specified face and its adjacent faces determines the directions of the face normal vectors. In Fig. 8, a designer changes dimension values $d 1$ and $d 3$. For the change from $d 3$ to $d 3$ ', the swept volume generated from the adjacent faces causes an interference.

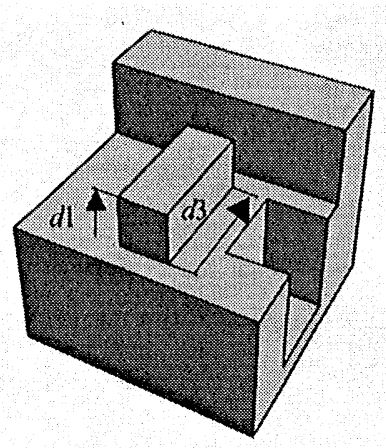

(a) product shape

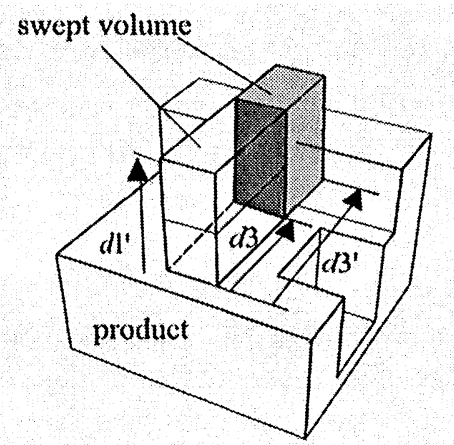

(b) swept volume

Figure 8: Swept volume by parameter change

When the specified face has faces which are dependent on it in the DFT, multiple swept volumes are generated according to the number of the faces, and they are combined before the interference calculation. Fig. 9 shows examples of multiple swept volumes. When one dimension is changed with two depended faces as shown in (a), multiple swept volumes are generated and combined according to $\Delta d$ (see (b), (c)). For the case (d) of the movement of a boss, two volumes are combined and additive and subtractive cells are generated (see (e) and (f)). 
If there is an interference between the swept volume and the product shape, the system asks a designer if he wants the change of the topology. If he permits the change, the system modifies the shape using the algorithm described in Section 2.

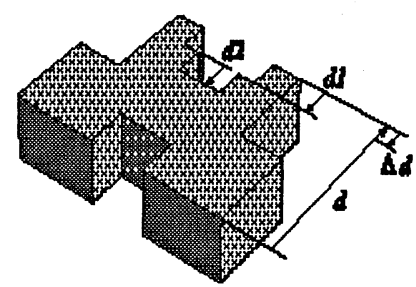

(a) Dependent Dimention

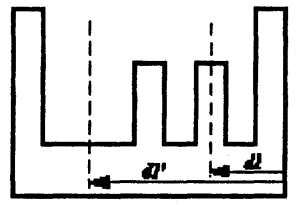

(d) Movement of 2 bost

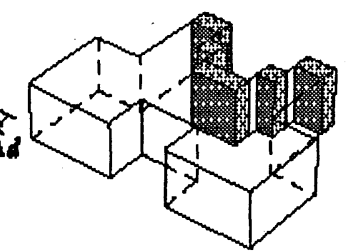

(b) $\Delta d<d I, d Z$

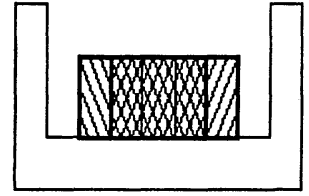

(c) two swopt valumes

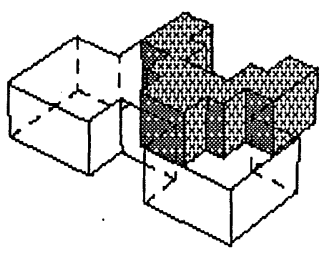

(c) $\Delta d>d I, d 2$

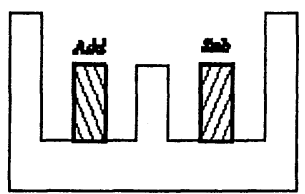

(f) combinod swept volum

Figure 9: Multiple swept volumes and their combination

\subsection{Change of feature types and geometrical constraints}

We modify the geometrical and topological constraints in the feature model according to the result of the interference calculation described above. The result reports deleted, divided, combined and newly generated faces with their IDs. Using this information, we modify the feature types and the DFT to maintain consistent relations among geometrical elements. When the modified feature does not satisfy the feature definition, we change its type, and when the dimension of the shape is over or under constrained, we detect this and change the constraints automatically or according to the designer's indication. 
Table 1: Classification of feature types according to a number of coincident faces

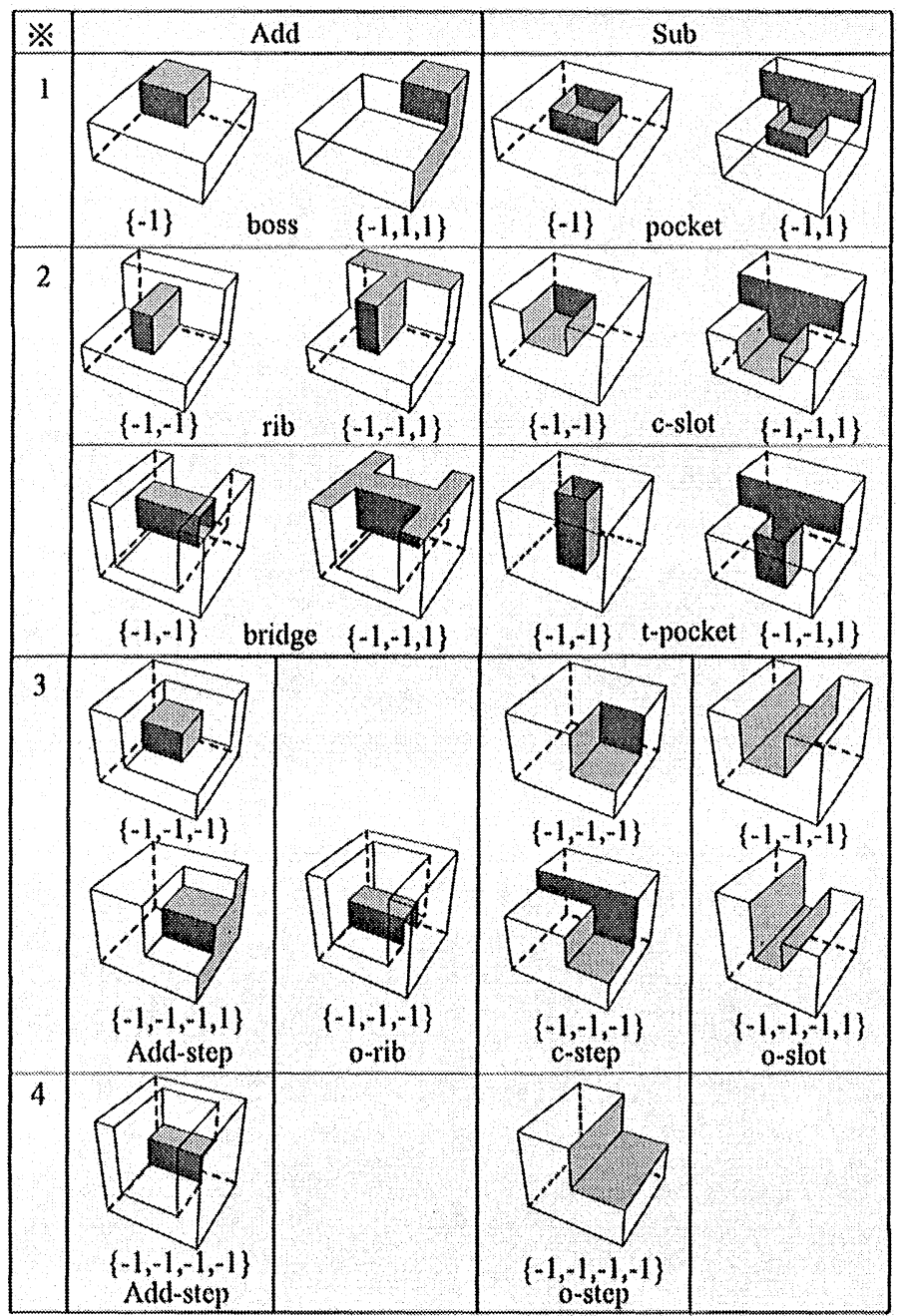

※ The Number of coincident faces with -1 or 0

When a face is deleted by the interference, the type of the feature to which the face belongs is changed according to the number of the coincident faces in the feature table. Table 1 shows classification of feature types. The feature type is classified by feature attribute $A d d / S u b$, the number of coincident faces which have coincidence attribute -1 and 0 , and the positions of coincident faces. For example, when a feature is additive and the number of coincident faces is 2 , the feature is a rib or a bridge according to whether the faces are adjacent or not. The number of the coincident faces with attribute 1 does not change a feature type, but the feature interferes with 
another feature sharing a common face. The interaction of feature has many varieties according to a number and positions of coincident faces, so typical examples are shown in Table 1.

We show two examples of feature changes in Figs. 10 and 11. In Fig. 10, the closed slot is changed into an open slot because one open face is added from the result that top face Block_U is divided into Block_U [1] and Block_U [2] and face $f 1_{-} B$ is deleted. Here, if the interference result reports a coincident face $\left(d \rightarrow d^{\prime}\right)$, it is added to the list of coincident faces with its face ID $\left(B L \_B\right)$, and if not $\left(d \rightarrow d^{\prime \prime}\right)$, the list with null face ID to show no explicit boundary face.
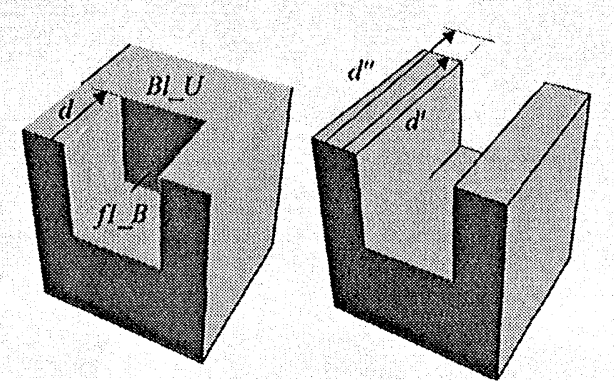

[Feature table

Dimension $=d$

fl: $c$-siot, sub,

$\{(B I, f I B, f l R, f I D\}$,

$\{-1,-1\}$

Dimensioned"

fl:o-siot, sub,

$\{f L, I R, f l D\}$

$(B] F, T T),(B I U, f I U)$

Figure 10: Change from closed slot to open slot
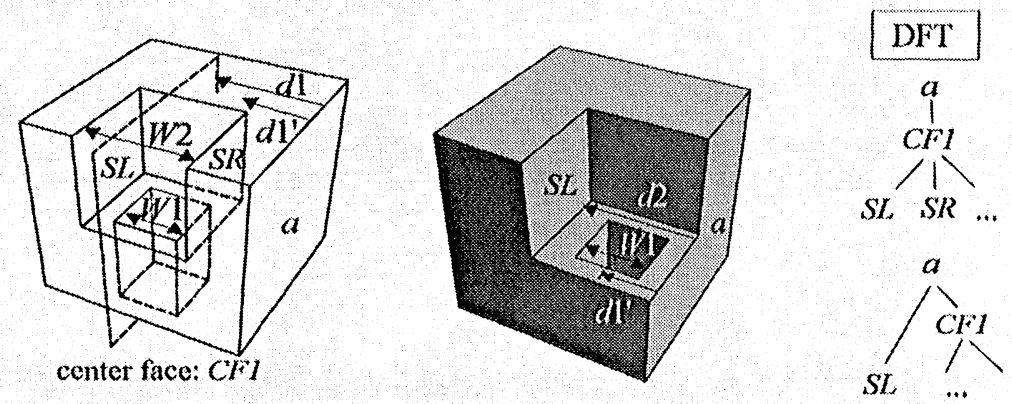

Figure 11: Change from closed slot to closed step

Along with feature maintenance, the geometrical constraints of dimensions are updated. When a face is deleted, combined or divided, the faces dependent on it should be changed to be dependent on a new face. The system detects these faces, and asks a designer to give them new dimensional constraints.

As shown in Fig. 11, when parameter value $d 1$ is changed to $d 1$ ', face $S R$ is deleted. Hence the feature is automatically changed from a closed slot to a closed step, and the dimensional parameter for face $S L$ is changed from 
width $W 2$ defined with a center face to distance $d 2$ from face $a$ according to the designer's indication. Then the DFT is changed as shown on the right in the figure.

\subsection{Examples}

We show some examples of product shapes generated by attachment of features and modified by changing dimensional parameters of the features. Fig. 12 (a) shows a product shape generated by attaching 11 features as shown in Figs. (b) and (c). Fig. (d) shows a modified shape without topological modification. Positions of features $f 3$ and $f 9$ are changed by indicating the distance to the center face along with a change of size, and side faces of feature $f l$ are slanted. Fig. (e) shows a shape with topological change. Feature $f 7$ is changed from a boss to a rib, and $f 6$ from a boss to a boss attached to the side faces.

Next example shown in Fig. 13 (a) contains many rib features. When the parameter $d 1$ allocating them is changed, multiple swept volumes are generated and one of them interferes with the product shape. If the designer wants to change this, the product is modified as shown in the figure (b).

All the constraints are maintained consistently as well as the shape modification, and they are reflected in the feature model.

\section{CONCLUSION}

We have proposed a method which enables efficient and unambiguous parametric modeling according to the designer's intention. The specification of the product indicated by the designer is represented symbolically in the product model as geometrical and topological constraints as well as a geometric model. When he changes a parameter, which is a dimension value of the geometrical constraint, the method checks whether it causes interference or not. If he wants the change, the method modifies the constraint information in the feature model according to the change of the geometric model. When it is ambiguous to determine the constraints automatically, the system asks the designer to indicate necessary information such as a datum face for a deleted face.

Through this study, we have obtained following conclusion.

Topological and geometrical constraints can represent the designer's intent and maintain validity of a feature model. 


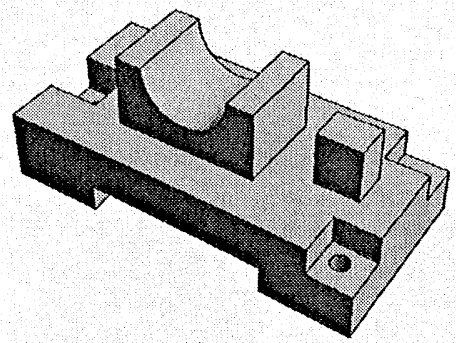

(a)

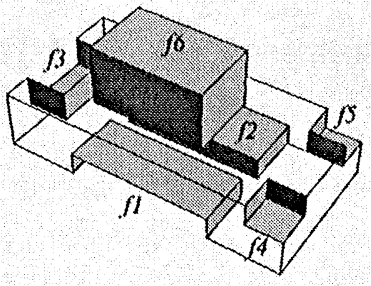

(b)

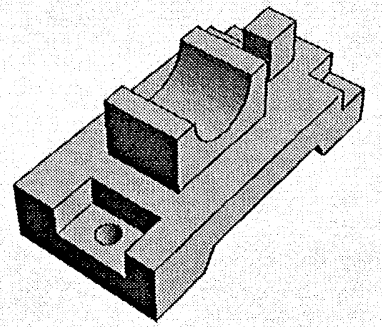

(d)

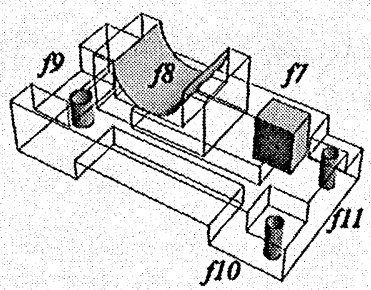

(c)

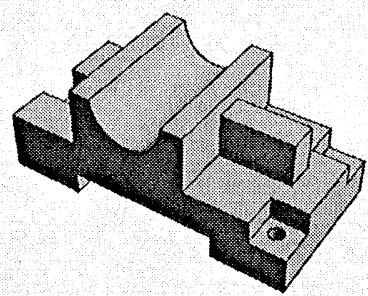

(e)

Figure 12: An example of a product shape

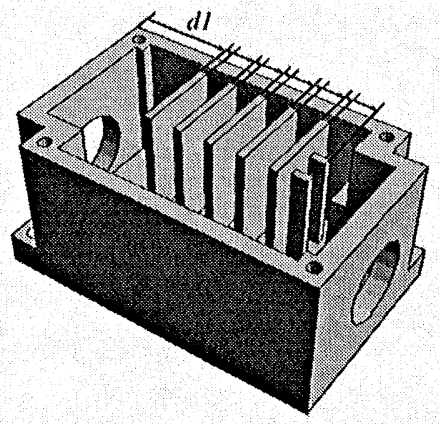

(a) Original shape

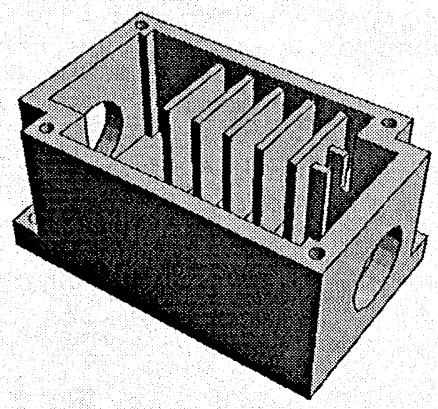

(b) Modified shape

Figure 13: An example of shape modification 
Direct modification of the feature model is realized without reevaluation of the feature operations by the interfere calculation between the product shape and the swept volume generated from movement of the specified faces.

This research is partly supported by the Grant-in-Aid for Scientific Research (C) from the Ministry of Education, Science, Sports and Culture, Japan.

\section{REFERENCES}

Bidarra, R., de Kraker J.K. and Bronsvoort, W.F. (1998). Representation and Management of Feature Information in a Cellular Model, Computer-aided Design, 30, 4, pp.301-313.

Bidarra, R. and Bronsvoort, W.F. (1999). Validity Maintenance of Semantic Feature Models, Proc. 5th ACM Symposium on Solid Modeling, Ann Arbor, MI, USA, pp.85-96.

Capoyleas, V., Chen X. and Hoffmann C.M. (1996). Generic Naming in Generative Constraint-Based Design, Computer-aided Design, 28, 1, pp.17-26.

Chen, X. and Hoffmann, C.M. (1995). Design Compilation for Feature-Based and Constraint CAD, Proc. 3rd ACM Symposium on Solid Modeling and Application, Salt Lake City, Utah, pp.13-19.

Chen, X. and Hoffmann, C.M. (1995). Toward Feature Attachment, Computer-aided Design, 27, 9, pp.695-702.

Chen, X. and Hoffmann, C.M. (1995). On Editability of Feature-Based Design, Computeraided Design, 27, 12, pp.905-914.

Kripac, J. (1995). A Mechanism for Persistently Naming Topological Entities in HistoryBased Parametric Solid Models (Toplogical ID System), Proc. 3rd ACM Solid Modeling, Salt Lake City, Utah, USA, pp.21-29.

Higashi, M., Torihara, F., Takeuchi, N., Sata, T., Saitoh, T. and Hosaka, M. (1995). FaceBased Data Structure and its Application to Robust Geometric Modeling, Proc. 3rd ACM Symposium on Solid Modeling and Application, Salt Lake City, Utah, pp.235-246.

Hoffmann, C.M. and Juan, R. (1993). E-rep -- An Editable, High-Level Representation for Geometric Design and Analysis, In P.R. Wilson, M.J. Wozny and M.J. Pratt (eds), Geometric Modeling for Product Realizaton, Elsevier Science Publishers B.V. (NorthHolland), pp.129-163.

Hoffmann, C.M. and Joan-Arinyo, R. (1998). On User-Defined Features, Computer-aided Design, 30, 5, pp.321-332.

Hosaka, M. and Sata, T. (1994). Integrated CAD/CAM System, Ohmsha, Tokyo, Japan (in Japanese).

Nakamura, H., Higahsi, M. and Hosaka, M. (1997). Robust Computation of Intersection Graph between Two Solids, Computer Graphics Forum, 16, 3, pp.C79-C88.

Raghothama, S. and Shaprito, V. (1999). Consistent Updates in Dual Representation Systems, Proc. 5th ACM Symposium on Solid Modeling, Ann Arbor, MI, USA, pp.65-75.

Rossignac, J.R. (1990). Issues on Feature-based Editing and Interrogation of Solid Models, Computer \& Graphics, 14, 2, pp.149-172.

Shah, J.J. and Mäntylä, M. (1995). Parametric and Feature-Based CAD/CAM: Concepts, Techniques and Applications, John Wiley \& Sons. 